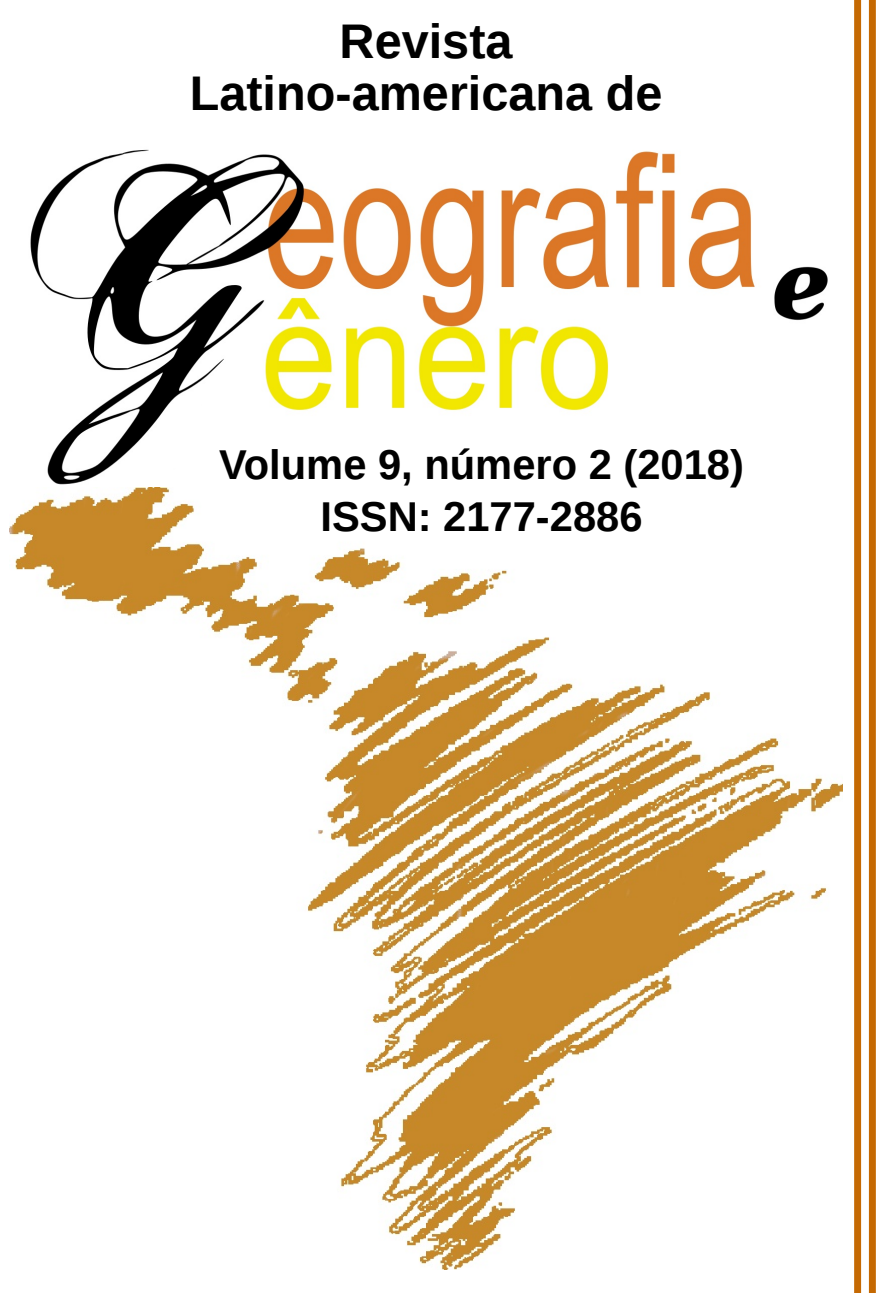

\title{
Las Series Web como \\ Cartografías de la Sexualidad: \\ La Representación de la \\ Interacción Lésbica en la \\ Ciudad Global en \\ Féminin/Féminin y The Foxy \\ Five
}

As Séries Web como Cartografias da Sexualidade: A Representação da Interação Lésbica na Cidade Global em Féminin/Féminin e The Foxy Five

The Web Series as Cartographies of Sexuality: The Representation of Lesbian Interaction in the Global City in Féminin / Féminin and The Foxy Five
Ana-Maria Cristea
Universidad de Oviedo - Espanha
cristeaana@uniovi.es

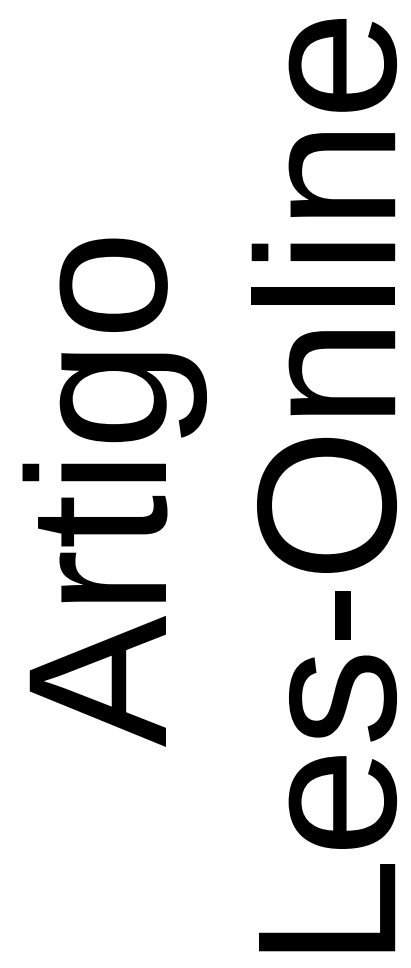

Como citar este artigo:

CRISTEA, Ana-Maria. Las Series Web como Cartografías de la Sexualidad: La Representación de la Interacción Lésbica en la Ciudad Global en Féminin/Féminin y The Foxy Five. Revista Latino Americana de Geografia e Gênero, v. 9, n. 2, p. 173188, 2018. ISSN 2177-2886.

Disponível em:

http://www.revistas2.uepg.br/index.php/rlagg 


\title{
Las Series Web como Cartografías de la Sexualidad: La Representación de la Interacción Lésbica en la Ciudad Global en Féminin/Féminin y The Foxy Five
}

\author{
As Séries Web como Cartografias da Sexualidade: A Representação da \\ Interação Lésbica na Cidade Global em Féminin/Féminin e The Foxy Five
}

\section{The Web Series as Cartographies of Sexuality: The Representation of Lesbian Interaction in the Global City in Féminin / Féminin and The Foxy Five}

\section{Resumen}

En el contexto del aumento de la visibilidad de personajes no heterosexuales en la cinematografía y en las series de televisión, como también de la cada vez más abundante literatura académica acerca del género y la sexualidad en la geografía, este artículo pretende examinar la serie para Internet como un formato de rápido crecimiento que desempeña un papel esencial en la representación y creación de espacios lésbicos. Con este propósito, analizaremos dos producciones - Féminin/Féminin (ROBICHAUD, 2014) y The Foxy Five (NEWMAN, 2016) - que se enfocan en la interacción entre personajes femeninos no heterosexuales en Montréal y Cape Town. Dichas series ofrecen una perspectiva compleja sobre las maneras en las que las mujeres lesbianas, bisexuales y trans (de diferentes grupos raciales) experimentan, cuestionan e intentan subvertir las geografías de poder.

Palabras-Clave: Serie Web; Identidad de Género; Lesbianas; Montréal; Cape Town.

\section{Resumo}

No contexto do aumento da visibilidade de personagens não heterossexuais em séries cinematográficas e de televisão, bem como na literatura acadêmica cada vez mais abundante sobre gênero e sexualidade na geografia, este artigo pretende examinar as séries para a Internet como um formato de rápido crescimento que desempenha um papel essencial na representação e criação de espaços lésbicas. Para isso, analisaremos duas produções - Féminin / Féminin (ROBICHAUD, 2014) e The Foxy Five (NEWMAN, 2016) - que se concentram na interação entre personagens não-heterossexuais em Montreal e Cape Town. Essas séries oferecem uma perspectiva complexa sobre as maneiras pelas quais as mulheres lésbicas, bissexuais e trans (de diferentes raças) experimentam, questionam e tentam subverter geografias de poder.

Palavras-Chave: Série Web; Identidade de Gênero; Lésbicas; Montreal; Cape Town. 
Las Series Web como Cartografías de la Sexualidad: La Representación de la

Interacción Lésbica en la Ciudad Global en Féminin/Féminin y The Foxy Five

\begin{abstract}
In the context of an increasing number of cinema and television productions focusing on or including non-heterosexual characters, as well as of the expanding scholarship surrounding gender, sexuality and space, this article explores the rapidly growing format of the web-series as sites of representation and creation of lesbian spaces. For this purpose, we will analyse two productions - Féminin/Féminin (ROBICHAUD, 2014) and The Foxy Five (NEWMAN, 2016) - which focus on non-heterosexual female interaction in Montréal and Cape Town. These series offer a complex perspective on the ways in which lesbian, bisexual and trans women (of different races) experience, question and attempt to disrupt geographies of power.
\end{abstract}

Keywords: Web series; Gender Identity; Lesbians; Montréal; Cape Town.

\title{
Introducción
}

El concepto de serie web hace referencia a "[t]odos aquellos seriales de ficción audiovisual creados para ser emitidos por Internet", que presentan características similares al medio televisivo (donde a veces se trasladan), pero que cuentan con una mayor accesibilidad e interactividad con los usuarios (MORANTE; HERNÁNDEZ, 2012, p. 142). En la última década ha surgido un número considerable de series para Internet que se centran en articulaciones de la diversidad de género y sexualidad. Por un lado, este aspecto se puede vincular con un fenómeno más amplio que supone el uso del Internet como plataforma para la creación de contenido queer y para fomentar una interacción más fácil y rápida entre miembros de la comunidad LGBT a través de redes específicas. Por otro lado, la proliferación de este género se puede interpretar como una crítica específica en cuanto a la ausencia o la representación errónea de la diversidad en el cine y en las series producidas para las grandes canales de televisión. ${ }^{1}$

Contrastado con diversas plataformas 'reales', el ciberespacio presenta ciertas ventajas para la comunidad LGBT, garantizadas por la relativa anonimidad de los usuarios y por proporcionar una vía de interacción con miembros y contenidos que muchas veces superan los límites físicos locales o nacionales, proporcionado un contexto para la construcción de 'una comunidad imaginaria gay'. Aunque no constituye una alternativa utópica de acceso total ${ }^{2}$, indudablemente forma parte de la esfera (semi)pública y/o contrapública, que, de modo parecido al cine, puede contribuir a la representación, formación, consumo y producción de identidades de género y sexuales.

1 Cabe mencionar que nuestro foco de interés, desde este punto de vista, no reside en los contenidos creados para plataformas de streaming como Netflix o Amazon, sino en las producciones independientes, de bajo presupuesto, accesibles de forma gratuita y que reivindican categorías normalmente excluidas de la representación audiovisual dominante. Mencionamos otros ejemplos relevantes como Studville TV (2013), centrado en personajes butch afroamericanos, o Two Jasperjohns (2011-2013) y Where the Bears Are (2012 presente), protagonizados por homosexuales que pertenecen a la comunidad de osos.

2 Por ejemplo, en su análisis de la distribución online de películas con temática lésbica, Rebecca Beirne $(2014 ; 2015)$ apunta al geobloqueo (Geoblocking), a los costes asociados a la compra y la facilidad de búsqueda como principales factores que limitan el acceso a dicho contenido (BEIRNE 2014; 2015). Este tipo de restricción se aplica, por ejemplo, en el caso de la segunda temporada de Féminin/Féminin, disponible en la plataforma Ici TOU.tv en Canadá y Studio 4 en Francia, cuyos contenidos no se pueden acceder desde otras zonas geográficas. 
Las Series Web como Cartografías de la Sexualidad: La Representación de la

Interacción Lésbica en la Ciudad Global en Féminin/Féminin y The Foxy Five

Este contexto plantea varias preguntas con respecto a la (no) distinción entre el espacio real y el espacio digital, las diferentes modalidades de representar la sexualidad y el género, la potencial reinscripción de la normatividad, la articulación de una dinámica local/global y sus potenciales efectos. En el presente artículo, nos centraremos en dos series creadas para Internet - la primera temporada de Féminin/Féminin (ROBICHAUD, 2015) y The Foxy Five (NEWMAN, 2016). Nuestro propósito es analizar las formas en las que dichas producciones proporcionan un enfoque crítico sobre la negociación del espacio (semi)público en Montréal y Cape Town, respectivamente, mientras intentan inscribir estos centros urbanos con un potencial lésbico que, aunque posicionado en un contexto global, conserva la especificidad cultural local. Dedicaremos la primera parte de nuestra investigación a la construcción del espacio público en relación con la presencia lésbica, que vincularemos posteriormente con la lucha para alcanzar la visibilidad como base de plataformas y comunidades generativas que sirven como punto de partida de estas producciones. A modo de conclusión, indicaremos una serie de puntos conflictivos y cuestionamientos.

\section{Metodología}

La metodología empleada en la presente investigación se basa en un análisis textual que incluye también referencias a los contextos socioculturales de cada una de las dos series, aplicando las pautas del análisis narrativo elaboradas por Sonja K. Foss (1996). La autora propone la selección de un artefacto que ilustre una dinámica de poder y centrarse en uno de sus aspectos específicos, de forma que el análisis revele ejemplos que subvierten las jerarquías existentes en un determinado contexto $y$, como resultado, que aluda o proponga formas alternativas de pensamiento y acción. Foss (1996) indica ocho elementos narrativos que pueden constituir el marco de la investigación: los personajes, el narrador, los acontecimientos, las relaciones temporales, las relaciones causales, el tema, el público y el espacio. Para el propósito de este artículo, el último aspecto enumerado resulta esencial, ya que las diferentes articulaciones y usos del espacio urbano reflejan el esfuerzo de las dos directoras de otorgar visibilidad a la presencia y a las experiencias de mujeres lesbianas, bisexuales y/o trans. Asimismo, aparte del enfoque textual, se ha realizado una recogida de información usando diferentes fuentes de bibliografía y webgrafía, que hacen referencia a una teorización general sobre el formato de las series web y el uso de los nuevos medios por la comunidad LGBTQI, a las dos producciones y/o a sus directoras, y, por último, al tema de las geografías lésbicas y queer, en general y aplicadas a las dos ciudades, Montréal y Cape Town.

El interés en las series web enfocadas en la diversidad sexual surgió en 2014, cuando, dentro del marco de una investigación doctoral sobre espacios extra-urbanos en el cine queer de Québec, descubrimos la obra de la directora Chloé Robichaud y el reciente estreno de Féminin/Féminin. Dicha serie se convirtió en 2015 en el objeto de una ponencia en el simposio Urban Encounters/Alternative Knowledges, organizado por el grupo de investigación Intersections/Intersecciones y celebrado el 22-25 de mayo en la Universidad de 
Las Series Web como Cartografías de la Sexualidad: La Representación de la

Interacción Lésbica en la Ciudad Global en Féminin/Féminin y The Foxy Five

Oviedo (España). Posteriormente, ampliamos el corpus, a través de una búsqueda y selección de series web que compartieran una preocupación por la intersección de la cultura urbana local y global y el deseo de crear un contenido representativo para la comunidad local, pero también relevante para otros contextos culturales. Somos conscientes de que la opción por un estudio comparativo resulta, en este caso, en un desequilibrio entre el espacio dedicado a la serie de Robichaud y el que alocamos a The Foxy Five, ya que en el primer caso hacemos referencia a varios capítulos de una temporada dedicada exclusivamente a la representación de personajes lésbicos, mientras que en el segundo caso sólo analizamos el único capítulo enfocado en las dos protagonistas no heterosexuales (el capítulo 4, titulado 'Femme Fatale and Lebo'). Además, también tenemos presentes las diferencias socioculturales esenciales que hay entre las dos ciudades en las que se ubican las protagonistas de las series. Sin embargo, nuestra investigación no pretende equiparar las realidades de una ciudad con las de la otra, ni generalizar las experiencias de una comunidad, sino que propone una línea de pensamiento transnacional que abre la posibilidad de dilucidar la interacción entre las fuerzas dinámicas globales y locales, aun más si incluye producciones en plataformas virtuales. Desde este punto de vista, nuestro estudio sigue, en parte, las propuestas de Jennifer Robinson (2011) y Kevin Ward (2010), quienes proponen un enfoque comparativo relacional, que permita cuestionar mecanismos que se dan dentro de una determinada ciudad a través de dinámicas similares de otros contextos.

\section{Breve Descripción de los Objetos del Análisis}

La serie Féminin/Féminin se enfoca en la vida cotidiana de seis mujeres lesbianas y bisexuales de Montréal y es el resultado de la colaboración entre la directora de cine Chloé Robichaud y Florence Gagnon, presidenta de la plataforma online lezspreadtheword.com. El primer capítulo de la primera temporada se estrenó a principios del año 2014, en la página de Gagnon, y, posteriormente, los siguientes siete capítulos fueron publicados en el sitio oficial de la serie, femininfeminin.com. La serie fue elogiada no sólo en Québec, donde recibió dos premios Gemeaux en 2015 (a la mejor serie web de ficción y la mejor actriz en una serie web), pero también a nivel internacional, siendo recomendada por páginas relevantes para la comunidad de mujeres lesbianas y bisexuales, como AfterEllen.com. Como consecuencia, tres años más tarde, se estrenó la segunda temporada en una plataforma canadiense de contenidos multimedia bajo demanda por Internet.

The Foxy Five es una serie web sudafricana que presenta los enfrentamientos de cinco mujeres de color de Cape Town con diferentes aspectos del sistema clasista, racista, sexista y transfóbico en el que viven. Jabu Nadia Newman, la realizadora de la serie, se inspiró en las mujeres que encabezaron la oleada de protestas estudiantiles, que surgieron en 2015 como crítica a las políticas de la 'Nación Arco Iris'3. Newman, que en aquel momento era estudiante en la Universidad de Cape Town, decidió renunciar a

3 La 'Nación Arco Iris' es un concepto implementado por el arzobispo Desmond Tutu en 1994 para describir la realidad sociocultural sudafricana, después de las primeras elecciones democráticas del país. 
Las Series Web como Cartografías de la Sexualidad: La Representación de la

Interacción Lésbica en la Ciudad Global en Féminin/Féminin y The Foxy Five

sus estudios y dedicarse a la creación de contenidos que se centraran en las diversas experiencias de las feministas negras de Sudáfrica. Como resultado, la serie The Foxy Five pretende proporcionar una perspectiva interseccional, presentando a través de cada uno de sus cinco personajes un determinado aspecto de la lucha feminista. De esta forma, Prolly Plebs encarna el feminismo hippy y la negociación racial, ya que es una mujer birracial. El personaje de Femme Fatale corresponde a una visión pro-sexo, que defiende el trabajo sexual y la fluidez del deseo. Blaq Beauty es la panafricanista y la principal representante de la lucha racial. Womxn We es la feminista intelectual, pero que también retrata la difícil realidad de los trastornos mentales. Por último, Unity Bond es la líder del grupo y, tal y como indica su nombre, es la que recopila todas las ideas de las otras mujeres, en un intento de mantener el equilibrio. La serie cuenta con seis capítulos que fueron grabados y publicados en el canal de YouTube The Foxy Five Tv desde junio de 2016 hasta junio de 2017, un proceso ralentizado por la recaudación paulatina de fondos a través de plataformas de crowdfunding.

\section{Reivindicaciones del Espacio Urbano}

El especial uso del espacio en las dos series seleccionadas y su relevancia en conexión con la articulación del deseo y la identidad lésbicos se debe considerar dentro del marco de las geografías de la sexualidad y concretamente de las geografías lésbicas. Dichos campos tienen su origen en los movimientos de emancipación gay y lesbiana y en la lucha feminista de los años 70, pero es a partir de los años 90 cuando se registra un auge de la publicación de trabajos teóricos pertenecientes al ámbito de la geografía cultural y social que se enfocan en el papel de la sexualidad en la percepción y experiencia de diversos espacios y las formas en las que el espacio y la sexualidad se constituyen mutuamente. Dentro de este contexto, se puede señalar el especial interés por los espacios urbanos y por la importancia de una cultura sexual pública, aspectos que se han criticado dentro de una línea de investigación que problematiza la percepción de los centros urbanos como indiscutibles espacios de liberación e invita a reconocer y a explorar un espectro más amplio de sexualidades y espacios. En un intento de implementar la teoría queer en la geografía, algunas autoras abogan por emplear el término queer como un posicionamiento que considera el género, la sexualidad y el espacio como categorías fluidas. De esta forma, lo que se pretende conseguir es un distanciamiento de los discursos previos que se basaban en la dicotomía homosexual/heterosexual y la aplicación de un marco interseccional que releve las tensiones y las contradicciones que surgen entre diferentes configuraciones de la identidad y subraye la exclusión y el control de ciertos cuerpos en espacios codificados como heteronormativos, pero también en ámbitos etiquetados como queer. Desde este punto de vista, cabe mencionar que la presente investigación sigue la línea propuesta por Kath Browne y Eduarda Ferreira $(2016$, p. 3) y emplea el término de 'lesbiana' como un concepto que se aplica a la sexualidad femenina o que se identifica como femenina, pero que implica una intersección de múltiples ejes de diferencia (cultural, social, racial, de clase etc.) y también una variación espacio-temporal. Por ende, la 
Las Series Web como Cartografías de la Sexualidad: La Representación de la

Interacción Lésbica en la Ciudad Global en Féminin/Féminin y The Foxy Five

insistencia en el carácter lésbico de las ubicaciones usadas en las dos series web responde a las identidades y prácticas expresadas directamente por las protagonistas, sin descartar, sin embargo, un posicionamiento queer en nuestro análisis.

Tal y como se ha señalado anteriormente, las dos series web están ambientadas en entornos urbanos e incluyen solo algunas breves referencias a imágenes del espacio extra-urbano ${ }^{4}$, reforzando en cierta medida los discursos teóricos que vinculan las sexualidades y los géneros disidentes con la ciudad. Consideramos relevante destacar el papel privilegiado que tiene la perspectiva lésbica en la serie de Robichaud, mientras que en el segundo ejemplo hay una clara insistencia en la necesidad de aplicar un enfoque anticolonial e interseccional, que incluye la presencia lésbica (the intersectional five/ las cinco interseccionales, como se autointitulan en el primer capítulo). En ambos contextos se puede identificar un esfuerzo común en cuanto a la codificación del espacio representado como marcado por una especificidad cultural (quebequense, sudafricana negra) en la que las mujeres tienen que negociar cuidadosamente su presencia y sus prácticas. En la escena inicial de Féminin/Féminin, se nos presenta un grupo de mujeres que están sentadas en la terraza de un bar y hablan de diferentes clichés. La llegada de Robichaud (que se interpreta a sí misma) perturba el contexto ficcional anterior. Las protagonistas establecen una conversación con ella y le piden que incluya a Suzanne Clément y Hélene Florent ${ }^{2}$ en la película que hará sobre ellas. Después de esta interacción con la misma directora, se inserta una presencia masculina estereotipada, cuyos comentarios sexistas - "dejadme adivinar quién es lesbiana y quién no", “„no os queréis dar un beso?” - son rechazados unánimamente con un gesto obsceno articulado por todas las mujeres del grupo. El intento de distinguir a las lesbianas de las heterosexuales falla rotundamente y lo que esta primera escena refleja, por lo tanto, es que en el universo en el que el público se incursiona lo que rige es la perspectiva y el deseo femeninos, mientras que la mirada masculina normativa no es sólo inaceptable, pero también ineficaz, ya que no puede leer los códigos de identidad que se emplean.

De forma similar, The Foxy Five comienza con una fuerte demanda de los derechos de las mujeres y un violento rechazo del acoso callejero. Las protagonistas responden de forma violenta a los comentarios por parte de un grupo de hombres $\mathrm{y}$, posteriormente, organizan una marcha en contra de la cultura del cat-calling y de la violación. El enfrentamiento con otro grupo de hombres durante la manifestación conduce a un diálogo cuyo propósito es, en definitiva, didáctico. Los personajes masculinos equiparan la exigencia de espacios públicos seguros con una defensa de 'putas y bolleras' y reciben como

4 El capítulo 4 de la primera temporada de Féminin/Féminin se centra en el personaje de Noémie, quien va de Blainville (un suburbio de Montréal) a la ciudad para visitar a su prima. Se presenta una transición de las conversaciones y actitudes incómodas manifestadas en su entorno inicial (extra-urbano) a un diálogo abierto con las protagonistas de la serie sobre la experiencia de ser lesbiana en Montréal. Esta contraposición refuerza, por consiguiente, la conceptualización de la ciudad como espacio idóneo para la articulación y exploración de la homosexualidad de uno/-a.

5 Dos actrices quebequenses muy conocidas. 
Las Series Web como Cartografías de la Sexualidad: La Representación de la

Interacción Lésbica en la Ciudad Global en Féminin/Féminin y The Foxy Five

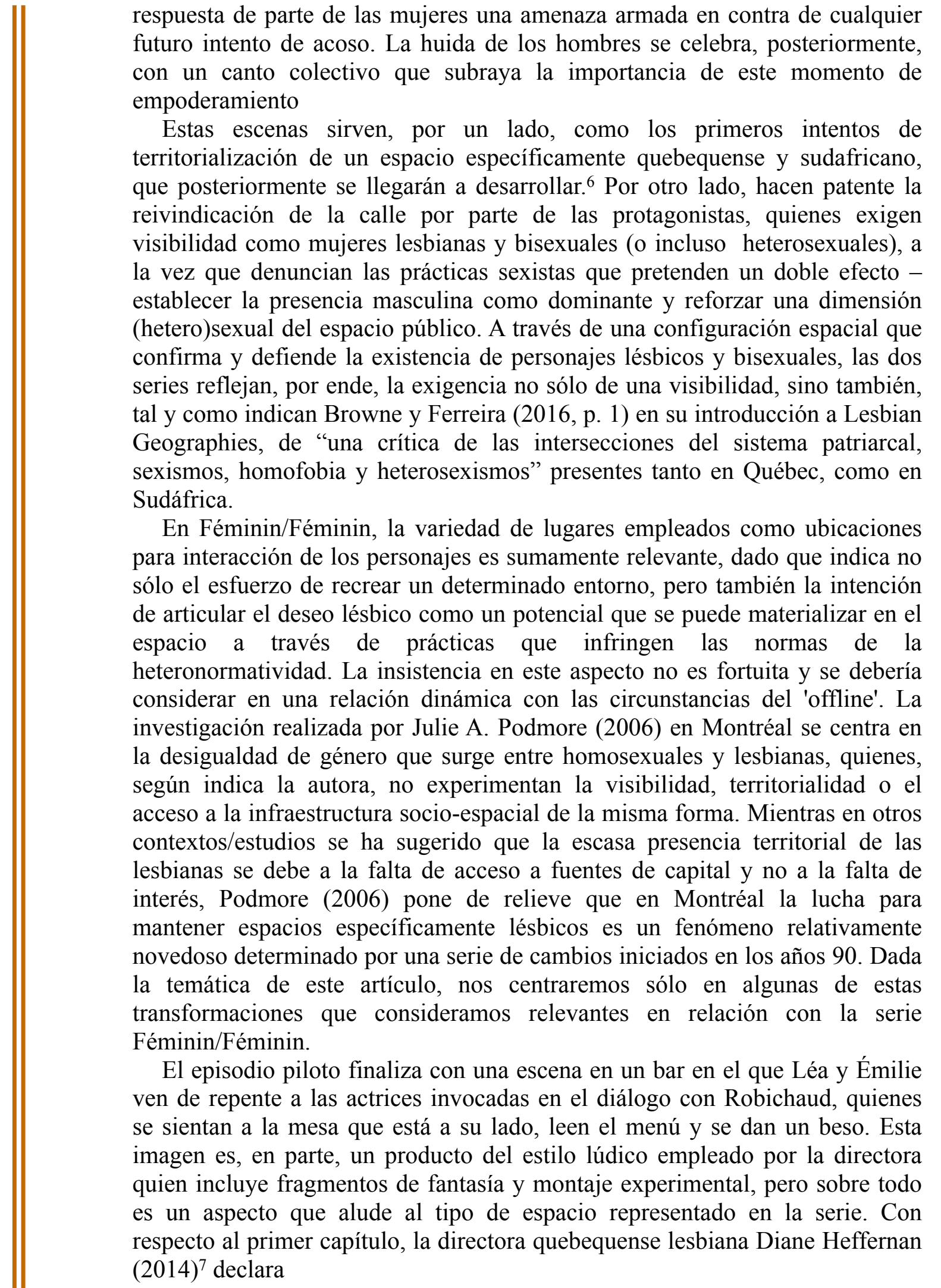

6 La territorialización sucede no sólo a nivel del espacio, sino también del idioma y de las referencias culturales, como es el caso de las dos actrices en Féminin/Féminin y el uso de canciones tradicionales en The Foxy Five. 
Las Series Web como Cartografías de la Sexualidad: La Representación de la

Interacción Lésbica en la Ciudad Global en Féminin/Féminin y The Foxy Five

Nos adentramos en los bares: música, baile, bebida, flirteo, cruising, etc. Estas escenas me evocaron recuerdos de cuando era joven. Me encantaba reunirme con grupos de lesbianas. ¡Me encantaba ir a los bares y ligar con chicas! Las lesbianas siempre han ido a los bares para conocer a otras lesbianas. Reviví mi juventud mientras veía el primer capítulo de Féminin/Féminin. (HEFFERNAN, 2014, s.p.).

Efectivamente, el período al que Heffernan hace referencia - los años 70-80 - fue etiquetado como la Edad de Oro de Montréal, marcada por la presencia de comunidades que contaban con sus propios territorios principalmente concentrados en el distrito (arrondissement) Le Plateau Mont Royal, que incluía agrupaciones institucionales y comerciales y un elevado número de bares de lesbianas. La importancia de la cultura de los bares, como también el tipo de interacción que ésta permitía entre mujeres lesbianas se ven claramente reflejadas en la serie de Robichaud, no sólo a través del uso de ubicaciones reales, con temática, pero también de forma más discreta, con algunas de las preguntas formuladas en los fragmentos del falso documental - '¿Beben las lesbianas más que otras mujeres?'. No es, sin embargo, una asociación que se limite sólo a la comunidad de lesbianas de Montréal o de Canadá, en general. Según Lee Wallace, el bar de lesbianas y el aula de clase son los dos espacios más privilegiados en el imaginario lésbico y, como consecuencia, son los más representados en las producciones audiovisuales con esta temática. ${ }^{8}$ De esta forma, Féminin/Féminin emplea un tema recurrente y reconocible para la comunidad de lesbianas - al menos, en el Norte global - y explora sus peculiaridades locales.

Uno de los aspectos más relevantes en cuanto al vínculo de la serie con la cultura de los bares es el uso del bar más famoso - y más fácil de reconocer por - el Royal Phoenix Bar. El Phoenix abrió en 2011 y se convirtió rápidamente en uno de los sitios de encuentro más importantes para las lesbianas de Montréal, constituyendo además, en dicho momento, el único bar regentado por una lesbiana. El mismo año que Robichaud estrenó la versión completa de su serie, el Phoenix hizo público que cerraría su actividad. La corta trayectoria de este bar se debe leer en el contexto de una reciente escasez de territorios lésbicos. Mientras la red casi utópica creada a lo largo de la Edad de Oro consiguió ampliarse hasta el principio de los años 90, a partir de 1992 estos espacios empezaron a reducirse de forma dramática. Aunque no exclusivamente, este fenómeno se debió al surgimiento de las políticas queer y las alianzas más fuertes entre lesbianas y homosexuales, que se materializó no sólo en la importación de grupos políticos como ACTUP, pero también en la organización de las primeras marchas del Orgullo y en la ampliación de las agendas de festivales gay y lésbicos con el propósito de incluir una mayor diversidad de identidades. Las nuevas zonas del Gay Village orientadas hacia

7 En 1987, la misma Heffernan en colaboración con Francine Aubin y Diana Bronson, dirigió un corto documental titulado Carnet de bar, en el que rendía homenaje a dos bares de la época destinados exclusivamente a lesbianas, el Lilith y el Labyris.

8 Cabe mencionar que la literatura mencionada por Wallace que recopila la importancia de los bares de lesbianas pertenece exclusivamente al ámbito angloamericano, por lo que no se debería considerar como un referente universal. 
Las Series Web como Cartografías de la Sexualidad: La Representación de la

Interacción Lésbica en la Ciudad Global en Féminin/Féminin y The Foxy Five

una clientela queer contrastaban con los pequeños bares de Le Plateau, la mayoría de los cuales pertenecían a mujeres feministas y lesbianas de habla francesa. Muchos de los establecimientos abrían grandes centros comerciales, contaban con una intensa promoción y se dirigían tanto a homosexuales como a lesbianas o al menos a mujeres más diversas a nivel de lenguaje, estética lésbica y etnia. Este cambio indicaba esencialmente que el distrito previamente dominado por la presencia gay estaba intentando responder a la demanda de un nuevo mercado que incluía una generación de mujeres que se identificaban como lesbianas y queer.

\section{La Experiencia Lésbica "Banal" como (Homo)Normatividad}

En Féminin/Féminin la mayoría de las ubicaciones utilizadas se pueden mapear en el antiguo Plateau, un detalle que se puede interpretar como un intento de rendir homenaje al núcleo lésbico tradicional de Montréal y de aludir a su continuidad en el presente (una genealogía que se puede intuir por el uso de imágenes antiguas de la ciudad y por la presencia de la lesbiana de edad mediana). Por otro lado, también podría subrayar el distanciamiento de los centros del Gay Village que están más bien orientados hacia el consumismo y que en muchos casos contribuyeron a una deterritorialización de la identidad lésbica a través de la creación de espacios mixtos. Pero aparte de (re)crear una cartografía, Robichaud (2014) también se propone engendrar una determinada conceptualización de la palabra 'lesbiana'. En una entrevista, Robichaud (2014, s.p.) explica que el aspecto más importante en la creación de la serie fue que "[a] menudo se nos habla de las consecuencias que tiene ser gay, de lo difícil que puede ser. Nosotras hemos querido realizar un programa para demonstrar que es cool, está bien, puede ser divertido". En otro artículo, la directora también insiste en su esfuerzo de alejarse de los estereotipos existentes y representar el lado 'cotidiano' o 'banal' de las vidas de las mujeres lesbianas de Montréal. Hay dos aspectos problemáticos que hemos identificado en este contexto. En primer lugar, el mero énfasis en la cultura de los bares, exenta del enfoque de la Edad de Oro en la construcción de la comunidad, presenta el riesgo de contribuir a una imagen específica de la cultura de salir y consumir, aunque no esté vinculada con el Village. En segundo lugar, el esfuerzo de 'banalizar' la homosexualidad femenina plantea importantes cuestiones en cuanto a las implicaciones y los compromisos que condicionan la visibilidad de identidades de género y sexuales no normativas y el paso de la perspectiva estereotipada a la 'normalidad'.

Consideramos relevante que el análisis de esta producción se haga, por lo tanto, teniendo en cuenta las circunstancias de la integración social exitosa de ciertas comunidades LGBT y su acceso a la condición de ciudadano/-a con derechos completos a base de una normalización conocida como homonormatividad, asociada a políticas neoliberales orientadas al crecimiento. Según Duggan (2002), la homonormatividad constituye:

una política que no cuestiona las creencias e instituciones heteronormativas dominantes, sino que las defiende y las mantiene mientras promete la posibilidad de un electorado gay desmovilizado y 
Las Series Web como Cartografías de la Sexualidad: La Representación de la

Interacción Lésbica en la Ciudad Global en Féminin/Féminin y The Foxy Five

una cultura gay privatizada y despolitizada, anclada en la domesticidad y el consumo (DUGGAN, 2002, p. 179).

Coincidimos con Brown (2008) en su crítica del uso excesivo de este concepto y la necesidad de considerar las prácticas alternativas que se pueden dar en las grandes ciudades occidentales, como también la relevancia de otros centros geográficos y de la experiencias LGBT en el ámbito no metropolitano. Sin embargo, en el caso de Féminin/Féminin, usamos el marco de la homonormatividad como instrumento de cuestionamiento del tipo de representación empleada justo en un discurso 'alternativo', pero que se centra en la principal ciudad gay-friendly de Québec y uno de los destinos turísticos más populares para la comunidad LGBT a nivel mundial.

Los medios audiovisuales contribuyen a los discursos de la 'normalización' y/o asimilación de lo no heterosexual al privilegiar la visibilidad sólo de determinadas narrativas y personajes, que de esta forma se consolidan como entidades y prácticas aceptables. Según Steven Seidman (2004), en los discursos dominantes, como el de Hollywood, este proceso se ve justificado por un esfuerzo aparentemente reparativo, que marcaría el paso de una conceptualización de la homosexualidad como enfermedad o monstruosidad a la 'normalidad'. Este último aspecto implica una igualdad moral y psicológica con los sujetos heterosexuales, pero también supone "ajustarse a la identidad de género, relacionar el sexo con el amor y con las relaciones de tipo matrimonio, defender los valores familiares, personificar el individualismo económico y exhibir orgullo nacional" (SEIDMAN, 2004, p. 133). La normalidad, por lo tanto, implica la renuncia a una movilización política y la aceptación y apropiación de las prácticas e instituciones asociadas a la heterosexualidad, sin cuestionar su legitimidad o su carácter 'natural'.

En Féminin/Féminin, estos aspectos se ven reflejados principalmente en los fragmentos del falso documental que realiza Robichaud con las protagonistas, quienes tienen que contestar a las preguntas de la directora que tocan diversos temas relevantes. La mezcla de ficción y falsa realidad contribuye a la creación de un espacio de debate en el que cada personaje analiza sus propias acciones e identidad. La voz que la directora le otorga a la experiencia lésbica no difiere mucho del discurso sobre la experiencia heterosexual (una frase que se repite en todas las entrevistas), ya que el matrimonio y la reproducción se presentan como opciones reales, no problemáticos para el futuro. El realismo y la normalidad a los que Robichaud alude en sus entrevistas se traducen, por lo tanto, como una perspectiva que la teórica Merri Lisa Johnson (2006, p. 119) criticó con respecto a una serie de televisión icónica para el colectivo lésbico (The L Word) y que consiste en una énfasis en la monogamia y su "adhesión sin sentido crítico a las normas de culturales de la vida en pareja", mi traducción. Efectivamente, Léa, el personaje que durante el primer capítulo insiste en que no quiere estar en una relación estable y hace esfuerzos para evitar comprometerse con varios personajes femeninos, acaba formando una pareja a finales de ese mismo capítulo. El caso de Émilie es aún más relevante, ya que aparece pasando por una separación que le deja sin hogar y sin mascota, sólo para reinstaurar de forma inmediata la domesticidad con una nueva pareja. Por último, mencionamos como un elemento relevante la falta de diversidad, 
Las Series Web como Cartografías de la Sexualidad: La Representación de la

Interacción Lésbica en la Ciudad Global en Féminin/Féminin y The Foxy Five

más allá de pequeñas variaciones (lesbiana, bisexual, heterosexual, francófona, anglófona). La historia del único personaje no blanco es excesivamente corta, no supone un posicionamiento político asociado a la diferencia racial y parece servir sólo como pretexto para introducir la sensibilización con respecto al cáncer de mama y para representar el trauma que experimenta su pareja estable, con la que, anteriormente, había decidido tener un hijo. Aunque no cuestionamos la relevancia de las problemáticas abordadas por Robichaud para las mujeres, en general, y las lesbianas, en particular, resulta evidente que los temas destacados tienen en común la afiliación a pautas de vida reconocibles principalmente por la audiencia heterosexual blanca.

En The Foxy Five, Newman mezcla el activismo político con una perspectiva compleja en cuanto a las tensiones e incongruencias inherentes dentro del círculo de las feministas. De esta forma, le asocia a cada protagonista un determinado discurso feminista y, como en Féminin/Féminin, cada capítulo desarrolla la historia de cada uno de estos personajes. A diferencia del planteamiento de Robichaud, la diversidad de las experiencias se invoca constantemente a través de la insistencia en la intereseccionalidad, indicando que cada individuo puede acabar siendo víctima de alguna forma de opresión, como consecuencia no sólo de su género y/o su sexualidad, pero también de su raza, clase, (dis)capacidad. El uso de este determinado marco se puede leer en el contexto del Apartheid que hizo que la identificación como no heterosexual implicara un desafío general de las identidades de raza, clase, género y sexual que el sistema pretendía imponerle a la sociedad sudafricana . Para el propósito de este artículo y sobre todo del tema de este segmento, nos centraremos sólo en dos de los personajes - Femme Fatale (feminista pro sex y lesbiana) y Lebo, su amiga transgénero.

Mientras las protagonistas de The Foxy Five se reúnen en varias ubicaciones seguras para debatir o celebrar diferentes temas (dentro o fuera de casa), el rasgo definitorio de cada una también determina el tipo de espacio que explora. En el caso de Femme Fatale, como en Féminin/Féminin, la sexualidad no normativa se vincula a la cultura de salir. Pero es precisamente este carácter transgresor y sus consecuencias lo que Newman decide problematizar creando un contexto en el que resulta evidente que Lebo es vulnerable a varios niveles de discriminación que Femme parece ignorar. Las miradas indiscretas del taxista que las llevaba al club hacen que Lebo se sienta incómoda, por lo que decide salir antes del coche e ir a un baño público para volver a afeitarse la cara, donde las protestas de una mujer blanca funcionan como una primera señal de las formas en las que la discriminación en contra de las personas trans se puede materializar como exclusión de ciertos espacios. La insistencia de Femme en la forma impecable en la que su amiga 'pasa' como mujer ('Literalmente, te ves más mujer que yo') resulta ser el producto de una absoluta falta de sensibilidad, ya que después lleva a Lebo a un bar con temática "noche de chicas", donde finalmente se ve obligada a pagar por la entrada porque el guardia se niega a reconocerla como mujer. Su humillación aumenta en el momento en el que, después de que Femme haya intentado besarle, Lebo se ve abandonada por una ex novia y se enfrenta a otro incidente en el baño que incluye violencia física. El capítulo acaba en un tono didáctico, con las protagonistas compartiendo ideas sobre la violencia patriarcal y las 
Las Series Web como Cartografías de la Sexualidad: La Representación de la

Interacción Lésbica en la Ciudad Global en Féminin/Féminin y The Foxy Five

perspectivas (pre)coloniales sobre el género. Lebo contribuye de forma esencial a esta conversación con un pequeño monólogo en el que denuncia, aparte de una discriminación violenta general en la vida pública, la fetichización por parte de Femme quien la reduce a un simple 'obsequio para masturbación intelectual'. La sentencia final - 'esta casa de muñecas que tenéis aquí, he terminado con esta casa de muñecas' - funciona como un recordatorio de la discriminación y la marginalización que sufren ciertas categorías incluso en los círculos feministas y/o LGBT, como resultado de prácticas de regulación y normalización. Lebo denuncia esencialmente la vacuidad de una actitud aparentemente inclusiva, lo que podría funcionar como un punto de partida para el cuestionamiento general de la representación de los personajes no normativos en los medios, incluso en los alternativos.

\section{La (De)Territorialización de la Diversidad}

La comparación de Féminin/Féminn con The L Word mencionada anteriormente es recurrente y apunta hacia el esfuerzo de consolidar $y$ contribuir a un imaginario más amplio, transnacional, a través de los medios digitales. En la página web de la revista francesa Télérama, se celebró el estreno de la serie en un artículo titulado "Féminin/Féminin, la serie lésbica más realista que The L Word" (LEHOUX, 2014). Robichaud misma cita la serie estadounidense en la mayoría de sus entrevistas, mientras que Florence Gagnon, la iniciadora del proyecto y la copropietaria de la página web lezpreadtheword.com menciona en un artículo que

Este año, se celebran 10 años de The L Word [...]. Pensamos que es la hora de apropiarse del tema, de crear algo con sabor quebequense. Hay una gran escasez de recursos para la comunidad de lesbianas. Nos hemos propuesto crear modelos positivos y un punto de referencia en internet. Eso ya existe en Europa y en Estados Unidos, pero no en Canadá [...] Es importante ocupar nuestro sitio, crear nuestra propia cultura lésbica aquí (LEHOUX, 2014, s.p.).

La alusión al espacio de la última sentencia se ve justificada por los aspectos que hemos mencionados anteriormente. La insistencia en una identidad lésbica invariablemente territorial presenta, sin embargo, algunos aspectos problemáticos. En un estudio enfocado en la interacción lésbica en el bulevar St.- Laurent de Montreal, Podmore (2006) indica una mayor tendencia de identificación con los negocios y lugares de socialización de dicha calle que con otras zonas asociadas a la comunidad LGBT/queer, lo que indica la existencia de otros factores que determinan la preferencia por ciertos espacios, aparte de la mera presencia de otras lesbianas. Para la autora, la manifestación de contactos 'accidentales' que superan los marcos de unos espacios destinados específicamente a las mujeres lesbianas y/o queer es esencial, ya que "socava la separación de los lugares de sociabilidad lésbica de los espacios cotidianos" PODMORE, 2006, p. 345, mi traducción). De esta forma, Podmore (2006) llama la atención a la necesidad de un análisis de las espacialidades lésbicas que aplique una perspectiva anti-esencialista (en el sentido de superar el binomio espacio heterosexual/espacio homosexual) y que no descarte las 
Las Series Web como Cartografías de la Sexualidad: La Representación de la

Interacción Lésbica en la Ciudad Global en Féminin/Féminin y The Foxy Five

formas en las que las mujeres lesbianas también 'crean' espacio a través de sus prácticas. Por otro lado, la teórica invoca la utilidad de un enfoque deterritorial que se centrase no tanto en la idea de 'visibilidad' (espacio lésbico visible), sino en los mecanismos que hacen que las lesbianas se hagan visibles entre ellas .

El internet se puede considerar como una de las formas por las que la interacción lésbica se materializa en la ausencia de un espacio físico. Para Christopher Pullen (2010), el ámbito virtual tiene el potencial de estimular nuevas formas de conectar para el colectivo LGBT, pero también corresponde a una posible disminución de la comunidad física. Lo ideal, argumenta Pullen (2010), es fomentar la reconstrucción de los espacios reales (también) a través del uso de los recursos que nos provee el Internet. Desde esta perspectiva, tanto Robichaud, Gagnon, como Newman, proponen a través de sus proyectos la creación de redes interactivas que unen el online con las espacialidades reales. Gagnon creó su página web en 2012 como respuesta a la falta de territorio lésbico en Montréal, que intentó superar también mediante la organización de noches benéficas en diferentes bares que no tenían temática lésbica (como, por ejemplo, 1 Apt 200 o Fitzroy, que también aparecen en la serie). Estos encuentros, junto con el uso de plataformas de crowdfunding (yoyomolo.com), hicieron que la producción de la serie web se materializara. Ésta, a su vez, adquirió una visibilidad inesperada en los medios nacionales e internacionales y en festivales y se puede ver en su página oficial, a través de Vimeo, y en la plataforma de streaming Ici TOU.tv. Se ha difundido en el canal de televisión France 4 y la segunda temporada se ha grabado para la cadena de televisión canadiense Ici ARTV, de esta forma contribuyendo a la construcción de un imaginario lésbico no sólo quebequense, pero francófono. Usando fuentes similares de crowdfunding y agradeciendo la colaboración de la 'diáspora digital', la serie web de Newman también traslada la lucha por la visibilidad de la calle al ámbito online. ${ }^{9}$ En este caso, hay una oposición explícita a las injusticias en contra de la comunidad negra de Sudáfrica que rinde un homenaje estético a las películas de blaxploitation de los años 70, de forma que crea un potencial de identificación y de colaboración a la producción que supera las fronteras de Sudáfrica y contribuye a un movimiento transnacional de mujeres negras.

\section{Conclusiones}

Concluimos el presente artículo con un repaso de los principales puntos abordados a lo largo de nuestro estudio y con una serie de consideraciones que podrían funcionar como punto de partida para el desarrollo de futuras investigaciones. En nuestra examinación de dos series web, hemos destacado la forma por las que las directoras reflejan la problemática de la reivindicación de las mujeres, especialmente no heterosexuales, del espacio público físico en Montreal y Cape Town. Se ha tenido en cuenta que incluso los objetos del

9 Newman hace referencias explícitas a las protestas Fees Must Fall, en contra del aumento de las matrículas para estudiantes en 2015, y Pretoria Girls High en 2016. En este último caso se trata de una escuela que inicialmente aceptaba sólo estudiantes blancos y que en la actualidad representa una institución que supuestamente admite la diversidad, pero que fue acusada en 2016 por discriminación en contra del alumnado negro. 
Las Series Web como Cartografías de la Sexualidad: La Representación de la

Interacción Lésbica en la Ciudad Global en Féminin/Féminin y The Foxy Five

presente artículo están vinculados a dos grandes ciudades consideradas como representativas desde el punto de vista del reconocimiento de los derechos LGBT y que son, por ende, dos destinos relevantes a nivel mundial para miembros de dicha comunidad y hemos insistido en reconocer en nuestra investigación la importancia del contexto dentro del análisis de productos culturales y de la resistencia a la universalización de una cultura LGBT. Por otro lado, hemos propuesto un enfoque crítico que ha puesto de relieve el potencial uso de una perspectiva normativa, incluso en producciones independientes y de acceso general, y hemos finalizado enfatizando el potencial de las plataformas online de crear redes y mecanismos de movilización que pudieran tener un impacto a nivel de los espacios físicos. En este contexto, señalamos la necesidad de explorar de forma más detallada los factores que determinan que ciertas series web pasen al formato televisivo o incluso cinematográfico ${ }^{10}$, las características de las culturas fandom construidas alrededor de estas producciones e identificar los instrumentos y los códigos empleados por las categorías menos representadas y más vulnerables (como es el caso de Lebo de The Foxy Five) para usar los medios digitales para producir narrativas e imágenes de sí mismas.

\section{Referências}

BEIRNE, R. New Queer Cinema 2.0? Lesbian-focused films and the internet. Screen, v. 55, n. 1, p. 129-138. 2014.

Piracy, Geoblocking, and Australian Access to Niche Independent Cinema. Popular Communication. v. 13, n. 1, p. 18-31. 2015.

BROWN, G. Urban (Homo)Sexualities: Ordinary Cities and Ordinary Sexualities. Geography Compass. v. 2, n.4, p. 1215-1231. 2008.

BROWNE, K.; FERREIRA, E. Introduction to Lesbian Geographies. In: BROWNE, K.; FERREIRA, E. (Eds.). Lesbian geographies: gender, place and power. New York: Routledge, 2016. p. 1-28.

DUGGAN, L. The new homonormativity: the sexual politics of neoliberalism. In: CASTRONOVO, R.; NELSON, D. D. (Eds.). Materializing democracy: toward a revitalized cultural politics. Duke University Press, 2002. p. 175-194.

FÉMININ/FÉMININ. Produção: Chloé Robichaud. Paris. 2014. Disponível em: $<$ http://femininfeminin.com/>

FOSS, S. K. Rhetorical criticism: exploration \&amp; practice. 2nd ed. Prospect Heights, IL: Waveland Press, 1996.

10 Como es el caso de Carmilla, una serie web canadiense que se estrenó en 2014 en YouTube y que se convertirá en película. 
Las Series Web como Cartografías de la Sexualidad: La Representación de la

Interacción Lésbica en la Ciudad Global en Féminin/Féminin y The Foxy Five

HEFFERNAN, D. Féminin/Féminin. 2014. Disponível em $<$ http://wowlgbt.com/english/2014/01/21/femininfeminin/>.

JOHNSON, M. L. 'L' Is for 'Long Term': Compulsory Monogamy on The L Word. In: AKASS, K.; MCCABE, J. (Eds.). Reading The L word: outing contemporary television. New York: I.B. Tauris, 2006. p. 115-137.

LEHOUX, V. "Féminin/Féminin", la série lesbienne plus réaliste que "The

L Word". 2014. Disponible em: < https://bit.ly/2UVYINw>

MORANTE, F. M.; HERNÁNDEZ, P. La webserie: convergencias y divergencias de un formato emergente de la narrativa en Red. Revista Comunicación, v. 10, n. 1, p. 140-149. 2012.

PODMORE, J. A. Lesbians in the Crowd: Gender, sexuality and visibility along Montréal's Boul. St-Laurent. Gender, Place \& Culture. v. 8, n. 4, 333-355. 2001.

Gone «underground»? Lesbian visibility and the consolidation of queer space in Montréal. Social \& Cultural Geography, v. 7, n. 4, p. 595-625. 2006.

PULLEN, C. Introduction. In: PULLEN, C.; COOPER, M. (Eds.). LGBT identity and online new media. New York: Routledge, 2010. p. 1-13.

ROBINSON, J. Cities in a World of Cities: The Comparative Gesture. International Journal of Urban and Regional Research, v. 35, n. 1, p. 1-23. 2011.

SEIDMAN, S. Beyond the closet: the transformation of gay and lesbian life. [S.1.] Routledge, 2004.

THE Foxy Five. Produção Jabu Nadia Newman. África do Sul. 2016

WARD, K. Towards a relational comparative approach to the study of cities.

Progress in Human Geography, v. 34, n. 4, p. 471-487. 2010.

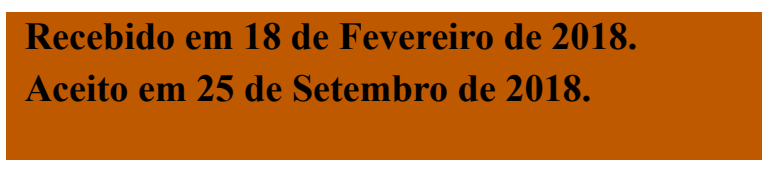

